\title{
Bioinformatics analysis to screen for critical genes between survived and non-survived patients with sepsis
}

\author{
YANFENG WU $^{1 *}$, LEI ZHANG ${ }^{2 *}$, YING ZHANG $^{3}$, YONG ZHEN $^{4}$ and SHOUYUE LIU ${ }^{5}$ \\ Departments of ${ }^{1}$ Pneumology, ${ }^{2}$ Neurology and ${ }^{3}$ Pediatrics, Second Hospital, Jilin University, Changchun, Jilin 130041; \\ ${ }^{4}$ Department of Neurosurgery, Northern Jiangsu People's Hospital, Yangzhou, Jiangsu 225001; ${ }^{5}$ Department of Neurosurgery, \\ Second Hospital, Jilin University, Changchun, Jilin 130041, P.R. China
}

Received February 18, 2017; Accepted August 24, 2017

DOI: $10.3892 / \mathrm{mmr} .2018 .9408$

\begin{abstract}
Sepsis is a systemic inflammatory response syndrome, which is mostly induced by infection in the lungs, the abdomen and the urinary tract. The present study is aimed to investigate the mechanisms of sepsis. Expression profile of E-MTAB-4421 (including leukocytes isolated from 207 survived and 58 non-survived patients with sepsis) and E-MTAB-4451 (including leukocytes isolated from 56 survived and 50 non-survived patients with sepsis) were downloaded from the European Bioinformatics Institute database. Based on the E-MTAB-4421 expression profile, several differentially expressed genes (DEGs) were identified and performed with hierarchical clustering analysis by the limma and pheatmap packages in R. Using the BioGRID database and Cytoscape software, a protein-protein interaction (PPI) network was constructed for the DEGs. Furthermore, module division and module annotation separately were conducted by the Mcode and BiNGO plugins in Cytoscape software. Additionally, the support vector machine (SVM) classifier was constructed by the SVM function of e1071 package in $\mathrm{R}$, and then verified using the dataset of E-MTAB-4451. A total of 384 DEGs were screened in the survival group. The PPI network was divided into 4 modules (modules A, B, C and D) involving 11 DEGs including microtubule-associated protein 1 light chain 3 alpha (MAP1LC3A), protein kinase C-alpha (PRKCA), metastasis associated 1 family member 3 (MTA3), and scribbled planar cell polarity protein (SCRIB). SCRIB and PRKCA in module B, as well as MAP1LC3A and MTA3 in module D, might function in sepsis through PPIs. Functional enrichment demonstrated that MAP1LC3A
\end{abstract}

Correspondence to: Dr Shouyue Liu, Department of Neurosurgery, Second Hospital, Jilin University, 218 Ziqiang Street, Changchun, Jilin 130041, P.R. China

E-mail: shouyueliu2016@hotmail.com

${ }^{*}$ Contributed equally

Key words: sepsis, differentially expressed genes, protein-protein interaction network, enrichment analysis, support vector machine in module D was enriched in autophagy vacuole assembly. Finally, the SVM classifier could correctly and effectively identify the samples in E-MTAB-4451. In conclusion, DEGs such as MAP1LC3A, PRKCA, MTA3 and SCRIB may be implicated in the progression of sepsis, and need further and more thorough confirmation.

\section{Introduction}

As a systemic inflammatory response syndrome (SIRS) induced by infection, sepsis is a life-threatening disease (1). The most common symptoms of sepsis are fever, confusion, and increased breathing and heart rate (2). Sepsis is usually caused by infection in the abdomen, the urinary tract and the lungs (3). Globally, sepsis accounts for a high mortality every year and results in the highest mortality in hospitals (4). Worldwide, the estimated incidence of sepsis is 18 million cases each year (5). In the United States, sepsis impacts $\sim 3$ in 1,000 people (6). In addition, severe sepsis results in $>200,000$ mortality incidences each year (7). Therefore, exploring the mechanisms of sepsis and developing novel therapies are necessary.

Callahan and Supinski demonstrated that downregulation of genes encoding important glycolytic and electron transport proteins help the development and maintenance of abnormalities in cellular energy metabolism in patients with sepsis (8). Nuclear factor-erythroid 2-related factor 2, a leucine zipper transcription factor that mediates stress response and redox balance, determines survival of sepsis patients through mounting a proper innate immune response $(9,10)$. Hypoxia-inducible factor $1 \alpha(\mathrm{HIF}-1 \alpha)$ in hypoxic and inflamed areas can release $\mathrm{T}$ cells that contribute to anti-bacterial response; thus, HIF-1 $\alpha$ in T cells may be used for therapeutic anti-pathogen strategies $(11,12)$. Several studies have reported that breast cancer 1, an important regulator of cell survival and DNA damage repair, can serve as therapeutic target for decreasing multiple-organ failure, systemic inflammation, and mortality in experimental sepsis (13-15). Sepsis and endotoxemia can cause declining B-cell CLL/lymphoma 2 (Bcl-2) levels in lymphocytes, and overexpression of lymphocyte $B c l-2$ has been proved to improve sepsis survival $(16,17)$. In 2016, Davenport et al (18) analyzed the transcriptomic response of 265 patients with sepsis in a discovery cohort, and screened 3080 differentially expressed genes (DEGs) in 
sepsis response signature 1 (including 820 upregulated and 2,260 downregulated genes) with a fold change $(\mathrm{FC})>1.5$ and adjusted $\mathrm{P}<0.05$. Using more strict thresholds, the present study investigated the DEGs between the survival and the non-survival group. In addition, protein-protein interaction (PPI) network and module analyses were conducted to identify key genes implicated in sepsis. Furthermore, a support vector machine (SVM) classifier was constructed and performed to further confirm the key genes identified.

\section{Materials and methods}

Expression profile data. Expression profiles of E-MTAB-4421 (used for the main analysis) and E-MTAB-4451 (used for the validation) were downloaded from the European Molecular Biology Laboratory-European Bioinformatics Institute database (www.ebi.ac.uk/arrayexpress/experiments), both of which were deposited by Davenport et al (18) and sequenced on the array of A-MEXP-2210-Illumina HumanHT-12_V4_0_ R1_15002873_B.E-MTAB-4421 included leukocytes isolated from 265 patients with sepsis (including 207 survivors and 58 non-survivors). Additionally, E-MTAB-4451 included leukocytes isolated from 106 patients with sepsis (including 56 survivors and 50 non-survivors). The patients were recruited from 29 intensive care units between Feb 1, 2006, and Feb 20, 2014. Following the admission of the patients, total blood leucocytes were rapidly isolated from whole blood samples $(\sim 10 \mathrm{ml})$ using the LeukoLOCK depletion filter technology (Thermo Fisher Scientific, Inc., Waltham, MA, USA) (18). The study of Davenport et al (18) was approved by national ethics committees and locally individual participating centers. In addition, the patients (aged $>18$ years) with sepsis caused by community-acquired pneumonia provided informed consent forms.

DEG screening. Probes corresponded to gene symbols were based on the annotation platform of Illumina HumanHT-12 V4. In addition, unloaded probes were filtered out. Gene expression value was obtained by calculating the mean value of the probes corresponded to the gene. Based on E-MTAB-4421, the DEGs between the survival group and the non-survival group were analyzed by the linear models for microarray data using R (limma package; www.r-project. org/) (19). Genes with $\mid \log \mathrm{FCl}>1$ and $\mathrm{P}<0.05$ were considered as DEGs. Using the Pheatmap package (cran.r-project.org/ web/packages/pheatmap/index.html) (20) in R, hierarchical clustering analysis was conducted for the DEGs.

PPI network analysis. The Biological General Repository for Interaction Datasets database (BioGRID, version BIOGRID-ORGANISM-3.4.135; www.thebiogrid.org) (21) which includes genetic and physical interactions, was utilized to map the identified DEGs into the human PPI network. Additionally, the non-DEGs which interacted with $\geq 10$ DEGs were also expanded into PPI network. The complete PPI network was constructed by the Cytoscape software (version 2.8; www.cytoscape.org) (22). In the PPI network, nodes and edges separately represented proteins and their interactions. Furthermore, the degree of a node was equal to the number of edges linked with it. Additionally, the Mcode (threshold: The degree of each node in module >2) (23) and
Table I. Top 10 differentially expressed genes with the smallest P-values.

\begin{tabular}{lcc}
\hline Gene & P-value & Log fold change \\
\hline SYNE1 & 0.000508 & -1.09577 \\
DSCR4 & 0.000603 & 1.049525 \\
GNB5 & 0.000691 & -1.09117 \\
KIAA1271 & 0.000875 & -1.07996 \\
LOC651643 & 0.001157 & -1.09987 \\
LOC730546 & 0.001244 & -1.09267 \\
KRT24 & 0.001267 & -1.08809 \\
LOC645445 & 0.001676 & 1.022562 \\
LOC650261 & 0.001911 & -1.11435 \\
UPF2 & 0.002267 & -1.0723 \\
\hline
\end{tabular}

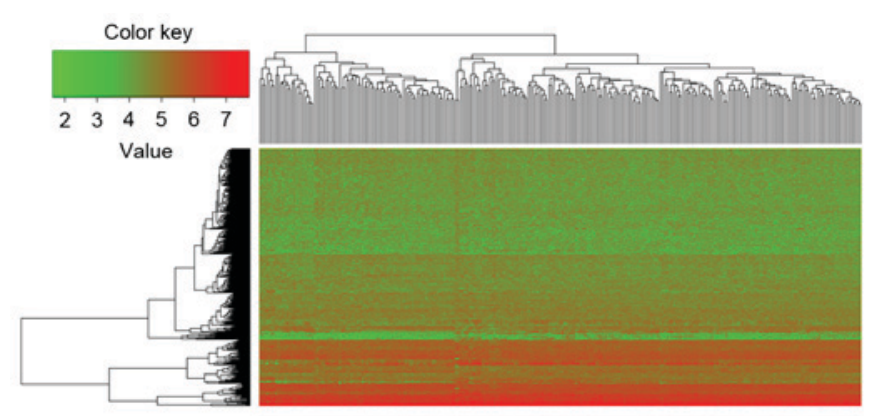

Figure 1. Heatmap of hierarchical clustering. Red and green colors represent high and low values, respectively.

BiNGO plugins (threshold, adjusted $\mathrm{P}<0.05$ ) (24) in the Cytoscape software were applied to perform module division and module annotation, respectively.

SVM classifier construction. Based on statistical theory, SVMs are effective classifiers, which can be applied in two-class classification problems of gene expression profiles and achieve high classification accuracy (25). Based on the expression values of key genes in the identified modules, the SVM function of e1071 package (version 1.6-7; cran.r-project.org/web/packages/e1071/index.html) (26) in $\mathrm{R}$ was used to confirm whether the key genes could distinguish between the two groups of samples (parameter: Gamma $=0.45$, cost $=5$ and cross $=10$ ).

Verification and assessment of the efficiency of SVM classifier. To verify the SVM classifier, the expression values of the key genes were extracted from E-MTAB-4451. In addition, the efficiency of SVM classifier was assessed by the sensitivity, specificity, and positive-(PPV) and negative predictive values (NPV) and the area under receiver operating characteristic curve (AUROC).

\section{Results}

DEGs analysis. Compared with the non-survival group, there were 384 DEGs in the survival group. Among these DEGs, 


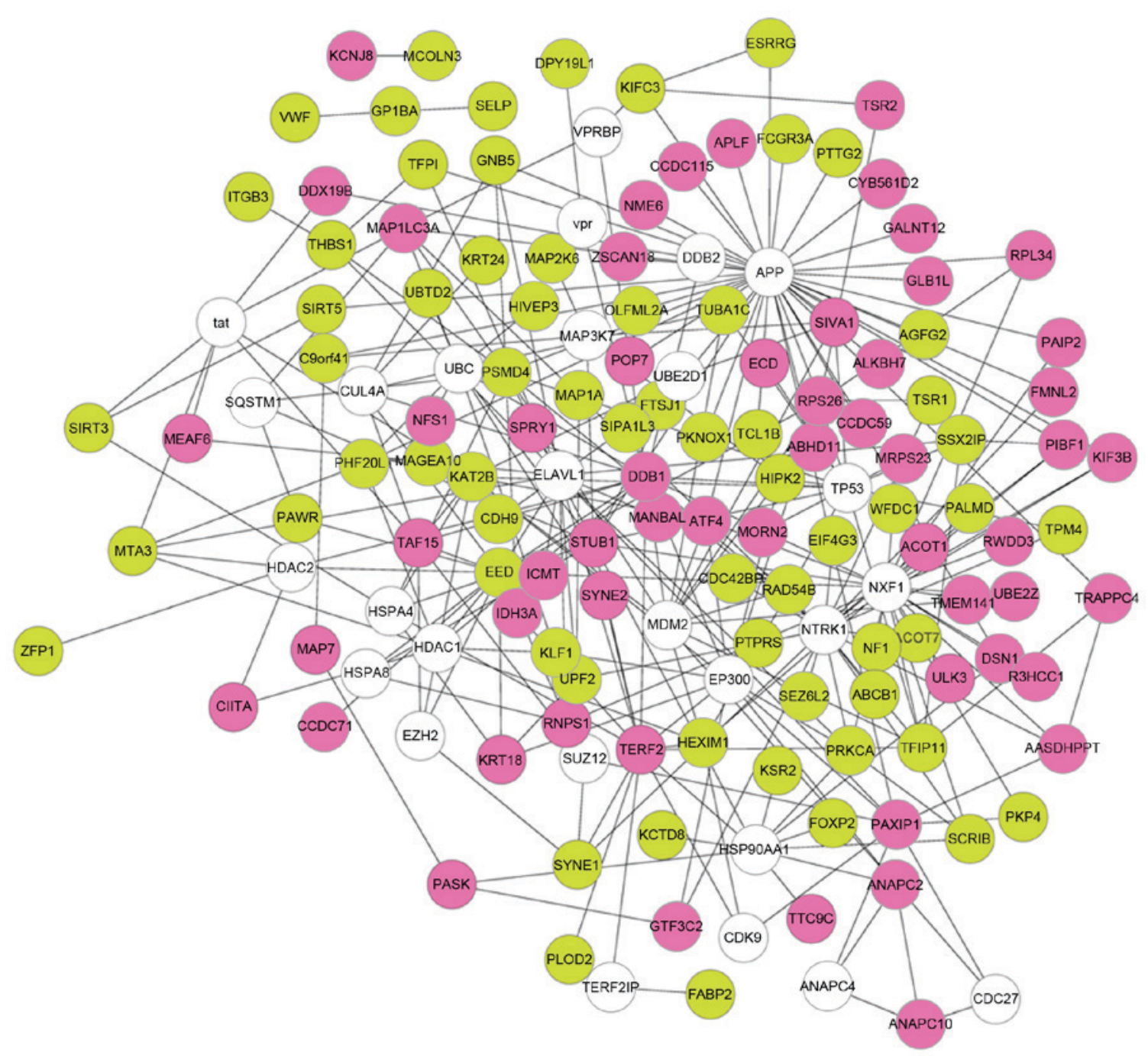

Figure 2. PPI network constructed for the DEGs. Pink and green nodes separately represent upregulated and downregulated genes, respectively. White nodes stand for the non-DEGs which interacted with $\geq 10$ DEGs and were expanded into the PPI network. PPI, protein-protein interaction; DEG, differentially expressed gene.

153 genes were significantly upregulated and 231 genes were significantly downregulated (Fig. 1). The top 10 DEGs with the smallest P-values are listed in Table I. Additionally, the heatmap of hierarchical clustering illustrated that the DEGs could distinguish the two groups of samples (Fig. 1).

PPI network analysis. A PPI network was constructed for the DEGs, which had 148 nodes and 305 interactions (Fig. 2). Based on the Mcode plugin, the PPI network was divided into 4 modules (module A, B, C and D; Fig. 3). A total of 11 DEGs [including microtubule-associated protein 1 light chain 3 alpha (MAP1LC3A), protein kinase C-alpha (PRKCA), metastasis associated 1 family member 3 (MTA3), and scribbled planar cell polarity protein (SCRIB)] were involved in the 4 modules, and almost all of them were among the top 21 in the PPI network according to degree rank (Table II). In addition, SCRIB and PRKCA in module B, as well as MAP1LC3A and MTA3 in module D hold interactions with each other. Using the BiNGO plugin, functional annotation was conducted for the 4 modules. A total of 9, 10, 12 and 5 functional terms were enriched for the DEGs in modules A, B, C and D, respectively. The main terms included ubiquitin-dependent protein catabolic
A
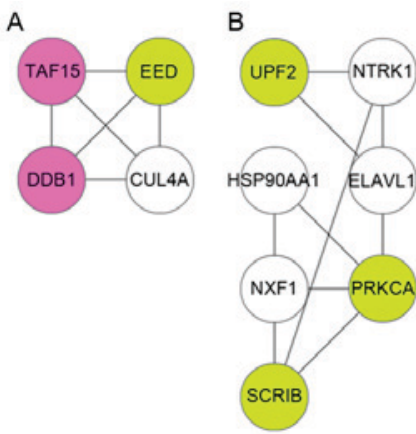

C

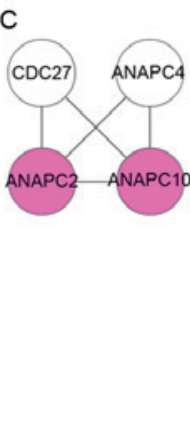

$\mathrm{D}$

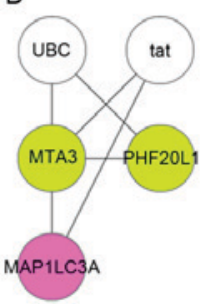

Figure 3. Modules A, B, C and D identified from the PPI network. Pink and green nodes separately represent upregulated and downregulated genes, respectively. White nodes stand for the non-DEGs which interacted with $\geq 10$ DEGs and were expanded into PPI network. PPI, protein-protein interaction; DEG, differentially expressed gene.

process (module $\mathrm{A}, \mathrm{P}=1.11 \mathrm{E}-03$ ), histone $\mathrm{H} 3-\mathrm{T} 6$ phosphorylation (module $\mathrm{B}, \mathrm{P}=9.78 \mathrm{E}-04$ ), regulation of mitosis (module $\mathrm{C}$, $\mathrm{P}=1.11 \mathrm{E}-03$ ), and autophagic vacuole assembly (module $\mathrm{D}$, $\mathrm{P}=1.11 \mathrm{E}-03$, which involved MAP1LC3A; Table III). 
Table II. Differentially expressed genes involved in the 4 modules identified from the protein-protein interaction network.

\begin{tabular}{lccccc}
\hline Gene & P-value & Log fold change & Degree & Degree rank in network & Module \\
\hline DDB1 & 0.025129 & 1.015005 & 15 & 1 & $\mathrm{~A}$ \\
EED & 0.016585 & -1.06699 & 14 & 2 & $\mathrm{~A}$ \\
MAP1LC3A & 0.037985 & 1.014367 & 7 & 10 & $\mathrm{D}$ \\
TAF15 & 0.037216 & 1.007017 & 7 & 10 & $\mathrm{~A}$ \\
ANAPC2 & 0.010137 & 1.022059 & 6 & 12 & $\mathrm{C}$ \\
PRKCA & 0.010663 & -1.04022 & 6 & 12 & $\mathrm{~B}$ \\
MTA3 & 0.035314 & -1.04998 & 5 & 15 & $\mathrm{D}$ \\
UPF2 & 0.002267 & -1.0723 & 5 & 21 & $\mathrm{~B}$ \\
PHF20L1 & 0.030279 & -1.15276 & 4 & 21 & $\mathrm{D}$ \\
SCRIB & 0.030139 & -1.07045 & 4 & 43 & $\mathrm{~B}$ \\
ANAPC10 & 0.022623 & 1.021597 & 3 & $\mathrm{C}$ \\
\hline
\end{tabular}

A

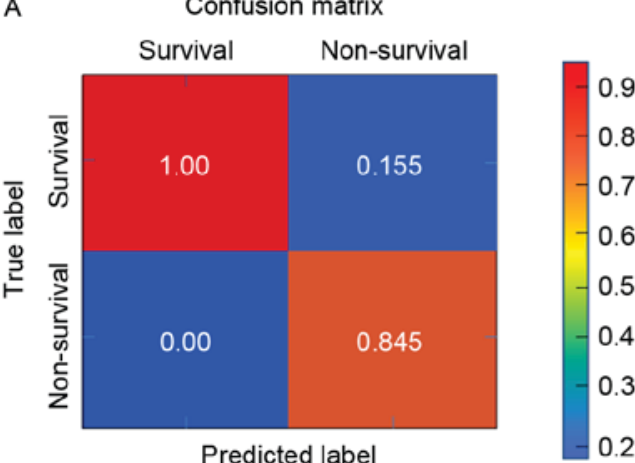

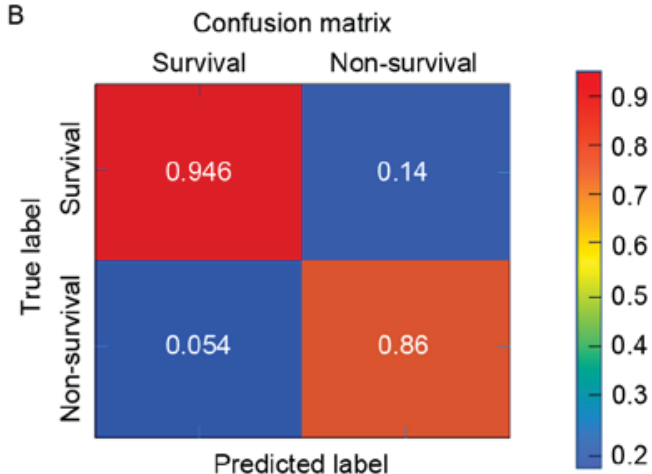

Figure 4. Confusion matrixes of sample identification for (A) E-MTAB-4421 and (B) E-MTAB-4451 using the SVM classifier. Red and blue indicate high and low accuracy, respectively. SVM, support vector machine; DEG, differentially expressed gene.

A

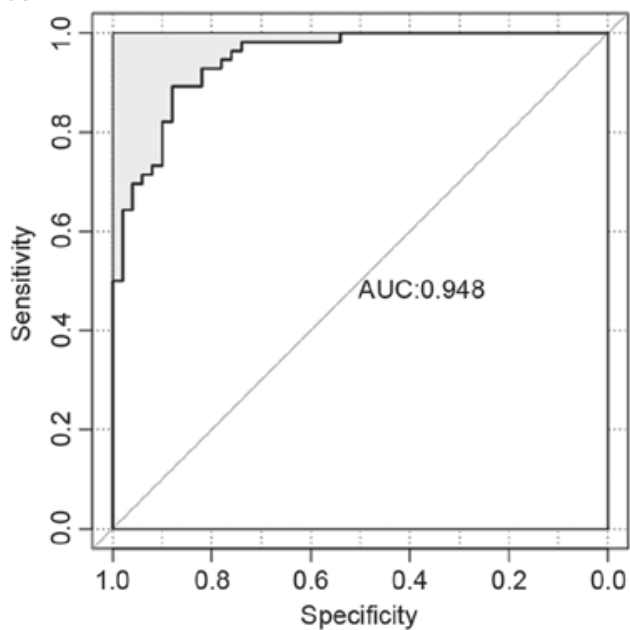

B

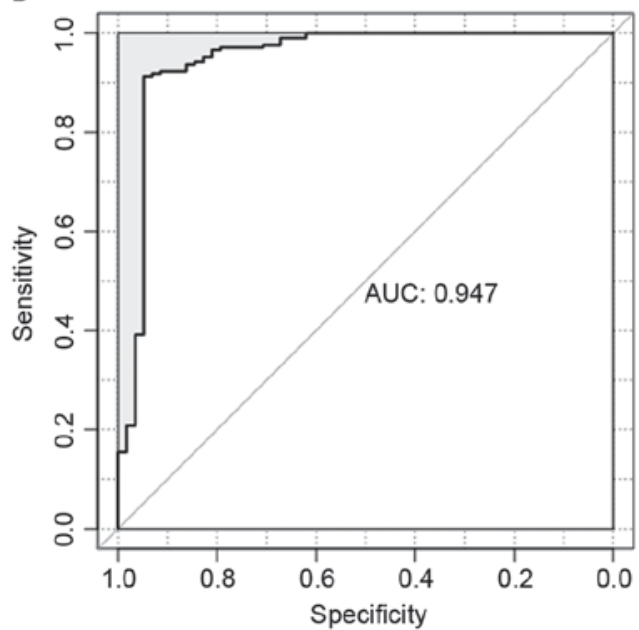

Figure 5. Receiver operating characteristic curves for (A) E-MTAB-4421 and (B) E-MTAB-4451. DEG, differentially expressed genes; AUROC, area under receiver operating characteristic curve.

SVM classifier construction. To confirm whether there was a difference in the key genes between the two groups of samples, the expression values of the 11 DEGs involved in the modules were extracted from E-MTAB-4421. Following this, an SVM classifier was constructed and its recognition capability to samples was observed. The results of the present study demonstrated that the SVM classifier could identify the survival samples (207/207; accuracy, 100\%) and the non-survival samples (49/57; accuracy, $84.5 \%$ ) with an overall accuracy of $96.6 \%$ (256/265; Fig. 4A). 
Table III. Functions enriched for the genes involved in the 4 modules.

\begin{tabular}{|c|c|c|c|c|}
\hline Module & Adjusted P-value & Gene number & Description & Gene symbol \\
\hline \multirow[t]{9}{*}{ Module A } & $2.07 \mathrm{E}-02$ & 2 & GO:6511 ubiquitin-dependent protein catabolic process & CUL4A, DDB1 \\
\hline & $2.07 \mathrm{E}-02$ & 2 & GO:19941 modification-dependent protein catabolic process & CUL4A, DDB1 \\
\hline & $2.07 \mathrm{E}-02$ & 2 & GO:6281 DNA repair & CUL4A, DDB1 \\
\hline & 2.07E-02 & 2 & GO:44257 cellular protein catabolic process & CUL4A, DDB1 \\
\hline & $2.93 \mathrm{E}-02$ & 1 & GO:718 nucleotide-excision repair, DNA damage removal & DDB 1 \\
\hline & $3.40 \mathrm{E}-02$ & 2 & GO:33554 cellular response to stress & CUL4A, DDB1 \\
\hline & 4.43E-02 & 2 & GO:6508 proteolysis & CUL4A, DDB1 \\
\hline & $4.58 \mathrm{E}-02$ & 2 & GO:44248 cellular catabolic process & CUL4A, DDB1 \\
\hline & $4.58 \mathrm{E}-02$ & 2 & GO:51704 multi-organism process & CUL4A, DDB1 \\
\hline \multirow[t]{10}{*}{ Module B } & 4.33E-02 & 1 & GO:35408 histone H3-T6 phosphorylation & PRKCA \\
\hline & 4.33E-02 & 1 & GO:35405 histone-threonine phosphorylation & PRKCA \\
\hline & 4.33E-02 & 1 & GO:46325 negative regulation of glucose import & PRKCA \\
\hline & 4.33E-02 & 1 & GO:51965 positive regulation of synaptogenesis & PRKCA \\
\hline & 4.33E-02 & 2 & GO:42330 taxis & PRKCA, SCRIB \\
\hline & 4.33E-02 & 2 & GO:6935 chemotaxis & PRKCA, SCRIB \\
\hline & 4.33E-02 & 1 & GO:32863 activation of Rac GTPase activity & SCRIB \\
\hline & 4.33E-02 & 1 & GO:60561 apoptosis involved in morphogenesis & SCRIB \\
\hline & 4.33E-02 & 1 & GO:1921 positive regulation of receptor recycling & SCRIB \\
\hline & 4.33E-02 & 1 & GO:35089 establishment of apical/basal cell polarity & SCRIB \\
\hline \multirow[t]{12}{*}{ Module C } & $2.52 \mathrm{E}-02$ & 1 & GO:7088 regulation of mitosis & ANAPC10 \\
\hline & $2.52 \mathrm{E}-02$ & 1 & GO:51783 regulation of nuclear division & ANAPC10 \\
\hline & 2.19E-03 & 1 & GO:8054 cyclin catabolic process & ANAPC2 \\
\hline & 7.21E-03 & 1 & GO:45773 positive regulation of axon extension & ANAPC2 \\
\hline & $8.55 \mathrm{E}-03$ & 1 & GO:48814 regulation of dendrite morphogenesis & ANAPC2 \\
\hline & $9.45 \mathrm{E}-03$ & 1 & GO:48639 positive regulation of developmental growth & ANAPC2 \\
\hline & $3.37 \mathrm{E}-02$ & 1 & GO:10720 positive regulation of cell development & ANAPC2 \\
\hline & $3.38 \mathrm{E}-02$ & 1 & GO:45927 positive regulation of growth & ANAPC2 \\
\hline & 4.13E-02 & 1 & GO:10975 regulation of neuron projection development & ANAPC2 \\
\hline & 4.89E-02 & 1 & GO:31344 regulation of cell projection organization & ANAPC2 \\
\hline & $9.69 \mathrm{E}-03$ & 2 & GO:51726 regulation of cell cycle & $\begin{array}{l}\text { ANAPC2, } \\
\text { ANAPC10 }\end{array}$ \\
\hline & $1.33 \mathrm{E}-02$ & 2 & GO:51128 regulation of cellular component organization & $\begin{array}{l}\text { ANAPC2, } \\
\text { ANAPC } 10\end{array}$ \\
\hline \multirow[t]{5}{*}{ Module D } & $3.15 \mathrm{E}-02$ & 1 & GO:45 autophagic vacuole assembly & MAP1LC3A \\
\hline & $3.15 \mathrm{E}-02$ & 1 & GO:16236 macroautophagy & MAP1LC3A \\
\hline & $4.65 \mathrm{E}-02$ & 1 & GO:6914 autophagy & MAP1LC3A \\
\hline & 4.65E-02 & 1 & GO:9267 cellular response to starvation & MAP1LC3A \\
\hline & $4.65 \mathrm{E}-02$ & 1 & GO:7033 vacuole organization & MAP1LC3A \\
\hline
\end{tabular}

GO, gene ontology.

Table IV. Efficiency of SVM classifier for E-MTAB-4421 and E-MTAB-4451 according to sensitivity, specificity, PPV, NPV and AUROC.

\begin{tabular}{lccccccc}
\hline Datasets & Number of samples & Accuracy & Sensitivity & Specificity & PPV & NPV & AUROC \\
\hline E-MTAB-4421 & 265 & 0.966 & 1 & 0.845 & 0.963 & 1 \\
E-MTAB-4451 & 106 & 0.906 & 0.946 & 0.86 & 0.883 & 0.935 & 0.947 \\
\hline
\end{tabular}

PPV, positive predictive value; NPV, negative predictive value; AUROC, area under receiver operating characteristic curve; SVM, support vector machine. 
Verification and assessment of the efficiency of the SVM classifier. E-MTAB-4451 was also downloaded from the EMBL-EBI database, which included leukocytes isolated from 56 survivors and 50 non-survivors. The expression values for the 11 DEGs were extracted from E-MTAB-4451 to further verify the SVM classifier. As a result, the SVM classifier could correctly identify 53 survival samples (53/56; accuracy, $94.6 \%$ ) and 43 non-survival samples (43/50; accuracy, $86 \%$ ) with an overall accuracy of $90.57 \%$ (96/106; Fig. 4B). Additionally, the efficiency of SVM classifier was high according to sensitivity, specificity, PPV, NPV and AUROC curve (Table IV and Fig. 5).

\section{Discussion}

In this study, a total of 384 DEGs (including 153 upregulated and 231 downregulated genes) were screened in the survival group. The PPI network constructed for the DEGs was divided into 4 modules, and they involved 11 DEGs (including MAP1LC3A, PRKCA, MTA3 and SCRIB). Additionally, a SVM classifier was constructed to investigate whether these 11 DEGs could distinguish between the two groups of samples, confirming that it could well recognize the survival from the non-survival samples with an overall accuracy of $96.6 \%$. Subsequently, the expression profile of E-MTAB-4451 was applied to verify the SVM classifier. The results illustrated that the SVM classifier could effectively identify the 53 survival and the 43 non-survival samples with an overall accuracy of $90.57 \%$.

SCRIB, which is overexpressed in endothelial cells and is essential for planar cell polarity and serves as a novel proinflammatory regulator in endothelial cells (27). Altman and Kong (28) demonstrated that protein kinase C proteins (PKCs) serve essential roles in human immune disorders, and can be used as therapeutic targets in several immune disorders including autoimmune diseases. For instance, PRKCA is the nonredundant and physiological PKC isotype in signaling pathways that are required for $\mathrm{T}$ cell-dependent interferon- $\gamma$ production and $\mathrm{IgG} 2 \mathrm{a} / 2 \mathrm{~b}$ antibody responses (29). Deficiency of PRKCB leads to defective B cell responses since B cells with PRKCB deficiency cannot activate the nuclear factor $(\mathrm{NF})-\kappa \mathrm{B}$ signaling pathway for $\mathrm{B}$ cell receptor (BCR), indicating that PRKCB has a critical role in BCR survival and may act as an important target for the treatment of B-lineage malignancies (30). In module B, SCRIB and PRKCA could interact with each other, indicating that they might hold roles in the progression of sepsis through this interaction.

The results of functional enrichment indicated that MAP1LC3A in module D was enriched in the autophagic vacuole assembly. Deficiency of autophagy-associated protein MAP1LC3B regulates the development of interleukin (IL)-17a-dependent lung pathology in the process of respiratory viral infection through endoplasmic reticulum stress-associated IL-1 (31). MTA3 is involved in the B lymphocyte transcriptional program and is a component in the $\mathrm{Mi} 2 /$ nucleosome remodeling and deacetylase (Mi2/NuRD) transcriptional corepressor complex $(32,33)$. In B cells, the complex can interact with the middle domain of Bcl-6 via MTA3 and negatively regulate several genes, including PR domain containing 1 with zinc finger domain through histone deacetylation activity $(34,35)$. Exogenous expression of MTA3-dependent
Bcl-6 in a plasma cell line results in reprogramming of cell fate, reactivation of the transcriptional program of B cell, suppression of the transcripts that are specific in plasma cells, and expression of surface markers of B lymphocyte cells (35). In module D, MAP1LC3A and MTA3 had interaction with each other, suggesting that MAP1LC3A and MTA3 might also function in the pathogenesis of sepsis via interaction.

In conclusion, based on the bioinformatics analysis of E-MTAB-4421,384 DEGs were identified in the survival group. In addition, MAP1LC3A, PRKCA, MTA3 and SCRIB might act in the progression of sepsis. However, further experimental investigation is required for these predictive results.

\section{References}

1. Singer M, Deutschman CS, Seymour CW, Shankar-Hari M, Annane D, Bauer M, Bellomo R, Bernard GR, Chiche JD, Coopersmith CM, et al: The third international consensus definitions for sepsis and septic shock (sepsis-3). JAMA 315: 801-810, 2016.

2. Hodgin KE and Moss M: The epidemiology of sepsis. Curr Pharm Des 14: 1833-1839, 2008.

3. Munford RS and Suffredini AF: Sepsis, severe sepsis and septic shock. In: Mandell, Douglas, and Bennett's Principles and Practice of Infectious Diseases. Bennett JE, Dolin R and Blaser MJ (eds). Elsevier Saunders, Philadelphia, PA, pp914-934, 2014.

4. Deutschman CS and Tracey KJ: Sepsis: Current dogma and new perspectives. Immunity 40: 463-475, 2014.

5. Lyle NH, Pena OM, Boyd JH and Hancock RE: Barriers to the effective treatment of sepsis: Antimicrobial agents, sepsis definitions, and host-directed therapies. Ann N Y Acad Sci 1323: 101-114, 2014.

6. Soong J and Soni N: Sepsis: Recognition and treatment. Clin Med (Lond) 12: 276-280, 2012.

7. Munford RS: Severe sepsis and septic shock. Harrisons Princip Int Med 16: 1606, 2005.

8. Callahan LA and Supinski GS: Downregulation of diaphragm electron transport chain and glycolytic enzyme gene expression in sepsis. J Appl Physiol (1985) 99: 1120-1126, 2005.

9. Thimmulappa RK, Lee H, Rangasamy T, Reddy SP, Yamamoto M, Kensler TW and Biswal S: Nrf2 is a critical regulator of the innate immune response and survival during experimental sepsis. J Clin Invest 116: 984-995, 2006.

10. Kong X, Thimmulappa R, Craciun F, Harvey C, Singh A, Kombairaju P, Reddy SP, Remick D and Biswal S: Enhancing Nrf2 pathway by disruption of Keap1 in myeloid leukocytes protects against sepsis. Am J Respir Crit Care Med 184: 928-938, 2011.

11. Thiel M, Caldwell CC, Kreth S, Kuboki S, Chen P, Smith P, Ohta A, Lentsch AB, Lukashev D and Sitkovsky MV: Targeted deletion of HIF-1alpha gene in T cells prevents their inhibition in hypoxic inflamed tissues and improves septic mice survival. PLoS One 2: e853, 2007.

12. Cramer T, Yamanishi Y, Clausen BE, Förster I, Pawlinski R, Mackman N, Haase VH, Jaenisch R, Corr M, Nizet V, et al: HIF-1alpha is essential for myeloid cell-mediated inflammation. Cell 112: 645-657, 2003.

13. Teoh H, Quan A, Creighton AK, Annie Bang KA, Singh KK, Shukla PC, Gupta N, Pan Y, Lovren F, Leong-Poi H, et al: BRCA1 gene therapy reduces systemic inflammatory response and multiple organ failure and improves survival in experimental sepsis. Gene Ther 20: 51-61, 2013.

14. Quan A, Creighton A, Bang K, Pan Y, Shukla P, Singh K, Al-Omran M, Lovren F, Verma S and Teoh H: Brcal gene therapy improves survival in experimental sepsis. In: Canadian Journal Of Cardiology 26: 146, 2010.

15. Ibrahim-Zada I, Lencinas A, Rhee PM, Maskaykina I and Friese RS: BRCA1 as a novel biomarker of Beta1-Blockade in sepsis. J Am Coll Surg 221: S40, 2015.

16. Iwata A, Stevenson VM, Minard A, Tasch M, Tupper J, Lagasse E, Weissman I, Harlan JM and Winn RK: Over-expression of Bcl-2 provides protection in septic mice by a trans effect. J Immunol 171: 3136-3141, 2003. 
17. OberholzerC,Oberholzer A,BahjatFR,MinterRM,TannahillCL, Abouhamze A, LaFace D, Hutchins B, Clare-Salzler MJ and Moldawer LL: Targeted adenovirus-induced expression of IL-10 decreases thymic apoptosis and improves survival in murine sepsis. Proc Natl Acad Sci USA 98: 11503-11508, 2001.

18. Davenport EE, Burnham KL, Radhakrishnan J, Humburg P, Hutton P, Mills TC, Rautanen A, Gordon AC, Garrard C, Hill AV, et al: Genomic landscape of the individual host response and outcomes in sepsis: A prospective cohort study. Lancet Respir Med 4: 259-271, 2016.

19. Smyth GK: Limma: Linear models for microarray data. In: Bioinformatics and computational biology solutions using R and Bioconductor Springer: pp397-420, 2005.

20. Kolde R and Kolde MR: Package 'pheatmap', 2015.

21. Stark C, Breitkreutz BJ, Reguly T, Boucher L, Breitkreutz A and Tyers M: BioGRID: A general repository for interaction datasets. Nucleic Acids Res 34 (Database Issue): D535-D539, 2006.

22. Kohl M, Wiese S and Warscheid B: Cytoscape: Software for visualization and analysis of biological networks. In: Data Mining in Proteomics Springer, pp291-303, 2011.

23. Bader GD and Hogue CW: An automated method for finding molecular complexes in large protein interaction networks. BMC Bioinformatics 4: 2, 2003.

24. Maere S, Heymans K and Kuiper M: BiNGO: A Cytoscape plugin to assess overrepresentation of gene ontology categories in biological networks. Bioinformatics 21: 3448-3449, 2005.

25. Ma S, Lv M, Deng F, Zhang X, Zhai H and Lv W: Predicting the ecotoxicity of ionic liquids towards Vibrio fischeri using genetic function approximation and least squares support vector machine. J Hazard Mater 283: 591-598, 2015

26. Dimitriadou E, Hornik K, Leisch F, Meyer D and Weingessel A: Misc functions of the Department of Statistics (e1071), TU Wien. R package 1: 5-24, 2008.

27. Kruse C, Kurz AR, Pálfi K, Humbert PO, Sperandio M, Brandes RP, Fork C and Michaelis UR: Polarity protein Scrib facilitates endothelial inflammatory signaling. Arterioscler Thromb Vasc Biol 35: 1954-1962, 2015
28. Altman A and Kong KF: Protein kinase $\mathrm{C}$ inhibitors for immune disorders. Drug Discov Today 19: 1217-1221, 2014.

29. Pfeifhofer C, Gruber T,Letschka T, Thuille N, Lutz-Nicoladoni C, Hermann-Kleiter N, Braun U, Leitges M and Baier G: Defective IgG2a/2b class switching in PKC alpha ${ }^{-/-}$mice. J Immunol 176: 6004-6011, 2006.

30. Su TT, Guo B, Kawakami Y, Sommer K, Chae K, Humphries LA, Kato RM, Kang S, Patrone L, Wall R, et al: PKC-beta controls I kappa B kinase lipid raft recruitment and activation in response to BCR signaling. Nat Immunol 3: 780-786, 2002.

31. Reed M, Morris SH, Owczarczyk AB and Lukacs NW: Deficiency of autophagy protein Map1-LC3b mediates IL-17dependent lung pathology during respiratory viral infection via ER stress-associated IL-1. Mucosal Immunol 8: 1118-1130, 2015.

32. Bowen NJ, Fujita N, Kajita M and Wade PA: Mi-2/NuRD: Multiple complexes for many purposes. Biochim Biophys Acta 1677: 52-57, 2004.

33. Fujita N, Jaye DL, Kajita M, Geigerman CS, Moreno CS and Wade PA: MTA3, a Mi-2/NuRD complex subunit, regulates an invasive growth pathway in breast cancer. Cell 113: 207-219, 2003.

34. Jaye D, Iqbal J, Fujita N, Geigerman CM, Li S, Karanam S, Fu K, Weisenburger DD, Chan WC, Moreno CS and Wade PA: The BCL6-associated transcriptional co-repressor, MTA3, is selectively expressed by germinal centre B cells and lymphomas of putative germinal centre derivation. J Pathol 213: 106-115, 2007.

35. Fujita N, Jaye DL, Geigerman C, Akyildiz A, Mooney MR, Boss JM and Wade PA: MTA3 and the Mi-2/NuRD complex regulate cell fate during B lymphocyte differentiation. Cell 119: 75-86, 2004.

This work is licensed under a Creative Commons Attribution-NonCommercial-NoDerivatives 4.0 International (CC BY-NC-ND 4.0) License. 